ISSN electrónico: 1885-5210

DOI: https://doi.org/10.14201/rmc202016e4555

\title{
EL BIENESTAR DE LOS UNIVERSITARIOS DURANTE EL CONFINAMIENTO POR COVID-19
}

\section{University Students' Wellness during COVID-19 Confinement}

\author{
Fernando GIL VILLAa; José David URCHAGA LITAGO ${ }^{\text {b }}$ Adrián SÁNCHEZ FERNÁNDEZa \\ aUniversidad de Salamanca. ' Universidad Pontificia de Salamanca (España). \\ Correo electrónico: gilvi@usam.es
}

Fecha de recepción: 11 de septiembre de 2020

Fecha de aceptación: 14 de septiembre de 2020

Fecha de publicación: 29 de enero de 2021

\section{Resumen}

El objetivo de este artículo es analizar los hábitos de los estudiantes universitarios durante el confinamiento que se produjo entre marzo y mayo de 2020 en relación al bienestar y el uso del tiempo libre. Para ello se han entrevistado a 911 de 43 universidades españolas entre mayo y junio del mismo año.

El sentimiento vital tira a la baja. La tristeza y la ansiedad parece haber hecho más mella en las mujeres. Aquellos que se han pasado el confinamiento en las grandes ciudades confiesan un mayor sentimiento de soledad. El consumo de alcohol más popular no parece haberse disparado durante la cuarentena -menos de un $10 \%$ la habría consumido casi a diario o a diario-. Tampoco el tabaco-el 82,2 \% no fuma-o el cannabis -el 92,3\% afirma no haberlo probado durante este periodo-.

El $69,7 \%$ ha leído un solo libro o ninguno. Como media, han visto 13,4 horas de series o películas a la carta, frente a 5 horas de informativos. De los datos se podría deducir cierta asociación, en la línea de otros estudios, entre el mayor consumo de alcohol y el mayor visionado de series. Quienes prefieren el género de "animación” parecen temer menos al virus que los que prefieren los documentales.

Palabras clave: COVID-19; bienestar; universitarios; tiempo libre; productos audiovisuales. 


\title{
EL BIENESTAR DE LOS UNIVERSITARIOS DURANTE EL CONFINAMIENTO POR COVID-19 \\ FERNANDO GIL VILLA; JOSÉ DAVID URCHAGA LITAGO; ADRIÁN SÁNCHEZ FERNÁNDEZ
}

\begin{abstract}
In this paper we will analyze the habits among university students during the confinement for Coronavirus, in relation to well-being and the use of free time. 911 students from 43 Spanish universities were interviewed between May and June of the same year. The vital feeling seems to be low. Sadness and anxiety is more evident in the case of women. Those who have spent the confinement in the big cities confess a greater feeling of loneliness. Alcohol consumption keeps in a low rate during the quarantine -less than $10 \%$ would have consumed it almost daily or daily. Neither do tobacco $-82.2 \%$ do not smoke- or cannabis $-92.3 \%$ would not tried it during this period. $69.7 \%$ have read a single book or none. On average, they have seen 13.4 hours of series or movies on demand, compared to 5 hours of news. From the data, a certain association could be deduced, in line with other studies, between higher alcohol consumption and higher series watching. Those who prefer "animation" contents seem to fear the virus less than those who prefer documentaries.
\end{abstract}

Keywords: COVID-19; wellness; university students; free time; audiovisual products.

\section{INTRODUCCIÓN}

La crisis desencadenada por la pandemia de COVID-19 se perfila en la historia de la humanidad como una de las más graves. El Director General de la OMS, ha declarado que pandemias tan devastadoras solo se producen una vez cada cien años y que sus efectos durarán décadas. Los efectos negativos en la economía ya superan los ocasionados por la Gran Recesión de 2008. No disponemos de antecedentes que nos permiten establecer comparaciones fiables. El confinamiento a que dio lugar el impacto de la primera ola no tiene precedentes en la duración y el carácter universal. De ahí la necesidad de estudios que analicen las consecuencias en todos los niveles. Si bien algunos autores han comentado el efecto negativo de la globalización en la epidemia, lo cierto es que también ha ayudado a enfrentarla de forma más rápida y eficaz, compartiendo información en tiempo real tanto entre los gestores y las ciudadanías. De la misma forma, la homogeneización del sistema de investigación académico, si bien tiene claras consecuencias negativas que hay que corregir, en casos excepcionales como este tiene una ventaja: tanto en la vertiente básica de la tecnología médica como en la de las ciencias sociales, da lugar a una sinergia que estudios que va a hacer que la crisis dure menos de lo habitual-se calcula que la mitad de la "gripe española" de hace un siglo- y sus efectos sociales sean analizados en un tiempo igualmente récord.

La presente aportación consiste en la obtención y análisis de información con la técnica de cuestionarios on-line, en este caso a la población específica de estudiantes universitarios españoles entre mayo y junio de 2020. La elección del momento es importante, porque al haberse terminado el Estado de Alarma la percepción de los encuestados cambia. Se tiene, en principio, una disposición más objetiva para evaluar el periodo traumático "superado". Nuestra intención era sondear algunos hábitos de los universitarios durante el confinamiento relacionados con su bienestar físico y psicológico centrándonos en el uso del tiempo. En concreto, dentro del tiempo no disponible, descartamos el dedicado a la obligación principal, que es el estudio y observamos parte del tiempo dedicado a las necesidades biológicas básicas (alimentación y sueño). Dentro del tiempo disponible, nos centramos en actividades voluntarias de carácter variado: social (voluntariados), no autotélicas (que no implican placer, como el ejercicio físico en cierto grado), tiempo libre desocupado (beber, fumar o ciertos visionados de pantallas), y ocio propiamente dicho (lectura y ciertos productos audiovisuales). 


\section{EL BIENESTAR DE LOS UNIVERSITARIOS DURANTE EL CONFINAMIENTO POR COVID-19 FERNANDO GIL VILLA; JOSÉ DAVID URCHAGA LITAGO; ADRIÁN SÁNCHEZ FERNÁNDEZ}

\section{MÉTODO}

Este estudio de investigación se llevó a cabo mediante una metodología cuantitativa en la que la recogida de datos se realizó a través de un cuestionario sociológico ad hoc tras el confinamiento provocado por la pandemia de COVID-19, del 31 de mayo al 27 de junio de 2020.

La divulgación del cuestionario se llevó a cabo a través de la red social Instagram, redirigiendo a los usuarios a completar el mismo a través de un enlace a Google Forms. La difusión del cuestionario se logró con la colaboración de la Sociedad Española De Excelencia Académica para llegar hasta los usuarios de asociaciones de estudiantes, facultades y delegaciones de alumnos de distintas universidades españolas; así como a la Organización Internacional de Juventud (OIJ), la Coordinadora de Representantes de Estudiantes de Universidades Públicas (CREUP) y otras agrupaciones juveniles con una alta representación de estudiantes universitarios.

Para conseguir un mayor alcance, se siguió también un muestreo por bola de nieve, solicitándose a los participantes que compartieran el cuestionario con otros compañeros universitarios. El uso de las redes sociales como instrumento para captar la muestra se justifica por su amplio alcance entre la población joven, entre la que se encuentran los individuos de interés para el estudio. Las encuestas son anónimas, la participación voluntaria y no se reporta ningún tipo de compensación por la realización de las mismas.

El cuestionario, que incluye varias preguntan identificativas (edad, sexo, universidad, curso académico, titulación y tamaño de la población de residencia durante el confinamiento), fue revisado previamente por tres expertos, y se realizó una prueba piloto sobre 10 estudiantes universitarios.

La muestra estuvo compuesta de 911 alumnos de 43 universidades españolas (77,4 \% mujeres; $22,6 \%$ varones). Con una media de edad de 21,9 años (DT: 4,8). Por curso, la mayoría eran de $10(28,8 \%)$, y en porcentajes similares de $2 \circ$, 3 o y último curso $(22,1 \%, 21,2 \%$ y $23,7 \%$ respectivamente) y un 4,3 \% de Máster.

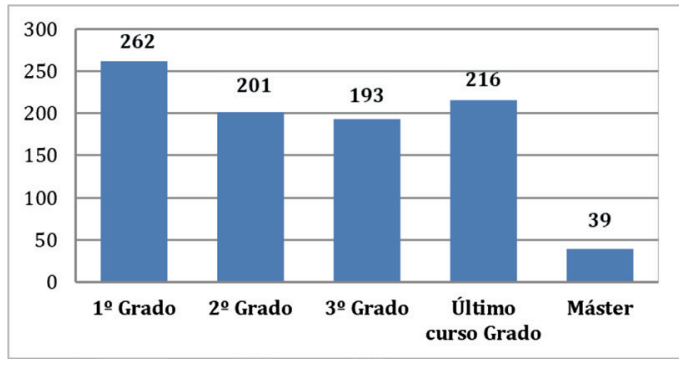

Gráfico 1. Composición de la muestra

\section{RESULTADOS}

\section{Bienestar}

Sentimiento vital, tristeza, soledad y ansiedad

Los estudios de revisión ponen de manifiesto las limitaciones de los trabajos que sirvan de referencia anteriores a la pandemia de $2020^{1}$. Los antecedentes sobre impactos psicológicos de situaciones comparables se centran en grupos específicos, como personal sanitario, o en zonas muy delimitadas y con características muy concretas, como en el caso del virus del Ébola. La cuestión por tanto es en qué medida el aumento de los días de confinamiento estricto, unido a la extensión universal, han repercutido en la proporción de los efectos consabidos: ansiedad, depresión, insomnio, aburrimiento, adicciones, obsesiones y fobias, etc.

Los primeros avances sobre la pandemia por COVID-19 muestran sobre entrevistados en 194 ciudades chinas durante la primera fase del confinamiento sufrido en aquel país pionero, que un $28,8 \%$ acusaron síntomas de ansiedad moderada o severa y un $8,1 \%$ niveles de estrés moderados o severos ${ }^{2}$. Vivir en áreas urbanas, en el domicilio familiar y gozar de estabilidad salarial mitigaría la probabilidad de sufrir ansiedad ${ }^{3}$. 


\section{EL BIENESTAR DE LOS UNIVERSITARIOS DURANTE EL CONFINAMIENTO POR COVID-19 FERNANDO GIL VILLA; JOSÉ DAVID URCHAGA LITAGO; ADRIÁN SÁNCHEZ FERNÁNDEZ}

Si nos fijamos en Italia, país vecino que sufrió, como España, uno de los impactos más duros de la crisis, encontramos datos para estadios más avanzados. En un cuestionario contestado por 2000 personas a finales de marzo y principios de abril de 2020 , se observó que el $57,1 \%$ confesaba dormir mal, un $41,8 \%$ malestar psicológico y un $32,1 \%$ síntomas de ansiedad ${ }^{4}$.

El impacto emocional de la pandemia ha podido ser registrado en las redes sociales. Por ejemplo, en plataformas chinas aumentaron las expresiones de ansiedad, depresión e indignación al tiempo que disminuían las que tiene que ver con emociones positivas y de satisfacción con la vida ${ }^{5}$.

En nuestro estudio, la puntuación general que ofrece la valoración general de la vida por el estudiante universitario tira a la baja, ya que es inferior a 6 en una escala de 0 a 10, siendo 10 la mejor vida posible subjetivamente percibida, y sin que haya diferencia significativa entre géneros ni entre cursos de grado.

Las mujeres muestran más tristeza y ansiedad que los varones, sin que sea significativa la diferencia en relación con la soledad. En las tres variables, las medias se sitúan cercanas al 2,5; siendo 2 poco y 3 bastante. Lo deseable sería que las puntuaciones medias fueran inferiores a 2 (poca tristeza, soledad o ansiedad).

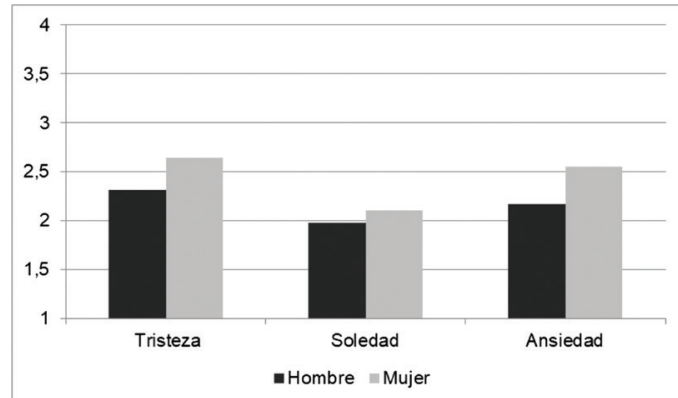

Gráfico 2. Tristeza, soledad y ansiedad por género

El condicionamiento espacial básico es importante a la hora de valorar la experiencia del confinamiento. En nuestro trabajo lo hemos medido por el número de personas con los que se comparte la vivienda por parte del estudiante encuestado. La gran mayoría $(81,7 \%)$ ha convivido con más de dos personas, un $15,9 \%$ con otra persona, y un $2,4 \%$ con nadie. Se advierte una relación significativa (Coeficiente de Contingencia, C: ,160; sig.: ,001) de la densidad residencial con el tamaño del núcleo población. Así, donde más se ha pasado en soledad el confinamiento es en el caso de estudiantes que se han quedado en las grandes ciudades: un 4,1 \% frente al $0,8 \%$ en poblaciones pequeñas (de 5.000 a 50.000 habitantes). Es posible que ello se deba a la dificultad para regresar a sus casas experimentada durante el confinamiento por una población típicamente flotante. Los universitarios que más han convivido con dos personas o más son los de poblaciones pequeñas ( $88 \%$ ), y los que menos, los de las grandes ciudades.

\section{Consumo de sustancias psicoactivas}

Si tomamos como punto de referencia los datos hechos públicos por el Ministerio de Agricultura, Pesca y Alimentación referidos a la semana del 6 al 12 de abril, vemos que las compras de cervezas aumentaron un $86,5 \%$, las de vino un $73,4 \%$ y los licores un $93,4 \%$ respecto a la misma semana de 2019. La cerveza es, por otra parte, el alcohol fermentado más popular entre las generaciones más jóvenes.

En general, los universitarios beben no tanto para olvidar las penas, como para divertirse; dentro de una pauta de consumo globalizada, la cual no está exenta de riesgos cuando en su variante más dura, la del atracón (Binge drinking). En un estudio entre universitarios españoles y canadienses, no se encontraron diferencias significativas entre los dos países ${ }^{6}$. Entre los motivos para consumir alcohol, pesan más los de refuerzo positivo que los de refuerzo negativo (para disminuir la ansiedad o la depresión). Los de animación son los que más pesan en el consumo elevado 


\section{EL BIENESTAR DE LOS UNIVERSITARIOS DURANTE EL CONFINAMIENTO POR COVID-19 FERNANDO GIL VILLA; JOSÉ DAVID URCHAGA LITAGO; ADRIÁN SÁNCHEZ FERNÁNDEZ}

"Por diversión", "Porque es emocionante", más que los sociales (celebración).

A conclusiones parecidas se ha llegado con jóvenes de 15 a 24 años: un $60 \%$ consume alcohol habitualmente el fin de semana. El $20 \%$ entre uno y dos litros. El $83 \%$ dice hacerlo porque facilita la diversión, el $77 \%$ porque facilita las relaciones sociales, y el $57 \%$ porque sirve para evadir problemas ${ }^{7}$.

En este sentido, ya se han llevado a cabo estudios en los que se evalúan los cambios en el consumo de alcohol y tabaco durante el confinamiento por COVID-198. El consumo de alcohol aumenta en algunos estudios, pero en otros disminuye tras situaciones de estrés postraumático. Apenas existe información sobre el confinamiento y solo se puede encontrar mención a algún precedente realizado sobre la anterior pandemia coronavírica de síndrome respiratorio agudo grave (SARS), en el que se detectó dependencia del alcohol para sanitarios incluso tres años después de haber trabajado. Por otro lado comprobamos que se insiste en la importancia de realizar estudios sobre el tema para evaluar el impacto emocional de la pandemia en el consumo de sustancias psicoactivas, especialmente la más comunes como el tabaco o el alcohol. Un estudio de proyección llevado a cabo por el grupo de salud pública del Well Being Trust, en EEUU, estimando una tasa de desempleo de tipo medio, entre 2020 y 2029, sugiere que se podrían dar hasta 75.000 muertes debidas a sobredosis de drogas y alcohol o suicidio como consecuencia del COVID-19. Principalmente por la incertidumbre económica y el estrés originado por el aislamiento ${ }^{\text {. }}$

Los datos aquí recabados abundan en la línea menos alarmista. El 82,2 \% de los entrevistados dice no fumar, sin diferencias significativas por género (Ji-Cuadrado, sig.: ,066), ni por curso
(Ji-cuadrado, sig.: , 279). El 64,7 \% no habría consumido cerveza durante el confinamiento. Solo un 7,4 \% lo habría hecho casi a diario o a diario. Las chicas habrían consumido algo menos que los chicos (C: ,125; sig.: ,002).

También se observa que, entre los estudiantes de grado, los que menos consumen son los de 10 (71\% nada), y los que más los de último curso $(55,6 \%$ nada) (C: ,148; sig.: ,022). Por último, el $92,3 \%$ afirma que no ha consumido cannabis durante el confinamiento, un 2,9\% lo habría hecho alguna vez al mes, un 2,3\% alguna vez a la semana y un $2,5 \%$ casi todos los días. No encontramos diferencias significativas ni por sexo (sig.: .062), ni por curso (sig.: ,510), aunque hay una tendencia a un menor consumo en mujeres $(93,8 \%$ no ha fumado, frente al $88,3 \%$ de los varones).

\section{Ejercicio físico}

El sedentarismo que implica el confinamiento puede alterar los hábitos alimentarios y físicos con consecuencias negativas para la salud. El aumento del tiempo de exposición a las pantallas constituye uno de los mayores riesgos, sobre todo en el caso de los jóvenes ${ }^{9}$. Considerando que, en general, la ingesta calórica suele aumentar cuando se gasta menos energía (entre un 35 y un $40 \%$ menos), algunos autores alertan de la "tormenta perfecta" que supondría, el confinamiento, unido a una gran variedad de canales de televisión bajo demanda, el aumento del tiempo libre, aburrimiento y mal humor, para un aumento futuro dramático de las enfermedades metabólicas ${ }^{10}$. Además se han detectado bajos niveles de ejercicio físico entre los jóvenes italianos y españoles de edad de 3 a 18 años ${ }^{11}$.

En nuestra aproximación, lo más frecuente $38,1 \%$ es no haber realizado nada o casi nada de ejercicio físico durante el confinamiento, en

a. https://wellbeingtrust.org/areas-of-focus/policy-and-advocacy/reports/projected-deaths-of-despair-during -covid-19/ 


\section{EL BIENESTAR DE LOS UNIVERSITARIOS DURANTE EL CONFINAMIENTO POR COVID-19 FERNANDO GIL VILLA; JOSÉ DAVID URCHAGA LITAGO; ADRIÁN SÁNCHEZ FERNÁNDEZ}

concreto un $38.1 \%$. Un $35,9 \%$ ha realizado entre 2 y 4 horas a la semana, y un $26 \%$ más de 4 . No hemos encontrado diferencias significativas ni por sexo ni por curso ( $\mathrm{p}$ : ,05).

Se da una correlación positiva entre la práctica de ejercicio y un sentimiento vital más positivo (Rho de Spearman: 134), negativa respecto al sentimiento de tristeza (Rho de Spearman: -,114), de soledad (Rho de Spearman: $-, 122)$ y de ansiedad (Rho de Spearman: -,164). Si bien el ejercicio no garantiza ni explica por sí solo un mayor bienestar psicológico general, el resultado no contradice la lógica de su influencia positiva.

\section{Tiempo libre}

Sobre una muestra de 2.000 jóvenes se ha observado que el $68,5 \%$, afirma ver series y películas en streaming o TV a la carta, siendo ese porcentaje superior en las mujeres $(72,2 \%)$. Un $41,5 \%$ consume videojuegos (un $47 \%$ si consideramos la franja de edad de 15 y 19). Un $36 \%$ consumiría televisión en general, un porcentaje parecido al que admite leer libros, revistas o cómics, o hacer deporte. Ir de tiendas lo practicaría un $30 \%$ (un 35,7 \% en el caso de las mujeres), y un $25 \%$ iría de bares o copas $(30,6 \%$ en la franja de 25 a 29 años) ${ }^{12}$.

Asistimos a la consolidación del uso de las tecnologías en el tiempo libre y ocio juvenil. Así, hace 25 años, estudios como el desarrollado en la Universidad de Oviedo por Rodríguez y Agulló, mostraban cómo la actividad más desarrollada entre los universitarios de Facultades y Escuelas técnicas era salir con amigos a bares y pubs ${ }^{13}$. El $77 \%$ admitían ver la televisión todos o casi todos los días, prefiriendo en primer lugar informativos y películas, y en solo en tercer lugar series. Mientras que las salidas nocturnas y el consumo de alcohol se han mantenido, el uso de pantallas ha aumentado como también el consumo de productos audiovisuales. Entre estos últimos, se imponen, por un lado, los contenidos de ficción de series o películas a la carta, y por otro los juegos. A su vez, dentro de estos, los más demandados son los videojuegos en general y los juegos de azar. Es necesario destacar también la asociación entre los videojuegos y las apuestas virtuales o presenciales ${ }^{12}$. Habrá que ver si futuros trabajos la ratifican, en cuyo caso estaríamos ante una de las principales derivaciones problemáticas del tiempo libre juvenil de factor tecnológico, junto con el del consumo de sustancias psicoactivas. De hecho, llama la atención el hecho de que el porcentaje de quienes practican los juegos de azar con mucha frecuencia es ya ligeramente mayor que el de hacer botellón (12,2 \% frente a $10,8 \%)^{12}$.

Por otra parte, en esa misma investigación se detecta que quienes comparten piso con otras personas, ven películas y series en streaming, y están "con amigos sin más" con más frecuencia, también salen más "de bares y copas", "a discotecas y a bailar" o "de botellón". Pues bien, en nuestro caso, también se da una correlación lineal positiva con el consumo de cerveza y el número de horas de visionado de series/películas (Rho Spearman: ,159; $p<, 001$ ). Estos últimos son también quienes más tarde se acuestan (rho: 170; $p<001)$.

En este punto, debemos considerar que los estudios epidemiológicos muestran la influencia negativa en la salud, incluyendo una predisposición al cáncer, de la ruptura continuada de los ritmos circadianos endógenos. Así, por ejemplo, los resultados del estudio MCC-Spain (con alrededor de 10.000 personas, entre enfermos de cáncer y grupo de control) señalan la importancia de la regularidad en el sueño y en la alimentación. En concreto, una cena temprana, así como mayores intervalos entre esta y el sueño, han podido observarse asociados con un menor riesgo de cáncer de mama y próstata ${ }^{14}$. En nuestro cuestionario no hemos descendido a un nivel de concreción que nos permitiera comprobar esta relación, pero hemos preguntado por las horas en 


\section{EL BIENESTAR DE LOS UNIVERSITARIOS DURANTE EL CONFINAMIENTO POR COVID-19 FERNANDO GIL VILLA; JOSÉ DAVID URCHAGA LITAGO; ADRIÁN SÁNCHEZ FERNÁNDEZ}

las que cenaban y se acostaban los universitarios durante el confinamiento. El 56,3\% acostaban a partir de la 1 en adelante. El 76,9\% cena en horario de 21 o 22 horas, un $13,3 \%$ entre las 23 y 24 , un $8,4 \%$ en el horario más temprano (19-20 h), y un $1,4 \%$ a partir de las $1 \mathrm{~h}$.

También se observa la tendencia al mayor consumo de productos audiovisuales ficticios de moda -o condicionados por la oferta de las grandes plataformas-. De hecho, podemos comparar la lectura con el consumo de contenidos audiovisuales. A un $20,4 \%$ de los universitarios no parece gustarles la primera, frente a un 15,7\%. La narrativa es el género preferido para ambos sexos, pero más para las chicas $(73,2 \%)$ que para los chicos $(66,0 \%)$, el ensayo es el segundo preferido por los chicos (12,6\%; chicas: 5,5\%), y el que menos la poesía ( $1 \%$; chicas: $5,5 \%$ ). Existen diferencias por curso que deben ser comentadas. Entre los alumnos de primer curso encontramos nada menos que un $21,8 \%$ que admite no gustarle la lectura, frente a un $11,6 \%$ del último curso. Este contraste, de confirmarse con otras muestras, sería compatible con los comentarios que aluden a los efectos negativos de las nuevas TIC en las habilidades de lectura y escritura en general, así como con las críticas al sistema educativo no universitario que señalan su incapacidad de inculcar la curiosidad intelectual en base a la lectura y el estudio. En todo caso, los datos obtenidos durante la experiencia del confinamiento son adecuados para corroborar la hipótesis de que la lectura no obligatoria no depende del tiempo libre, sino que es una es opción personal ${ }^{15}$ frente a interpretaciones opuestas $^{13}$.

Ahora bien, si lo habitual es haber leído un libro o ninguno $(69,7 \%)$, los universitarios entrevistados calculan que han visto a la semana 5,1 horas de informativos (DT: 5,02), por 13,4 horas de series o de películas (DT: 11,9), sin que se aprecien hay diferencias según sexo ni curso.

En cuanto al consumo por género del contenido visionado, no se han encontrado diferencias por curso, pero sí por género ( $\mathrm{C}: 169 ; \mathrm{p}<.001)$. Los hombres prefieren el contenido de acción (33\%; mujeres: 17,6\%), mientras que para las mujeres se inclinan por la comedia $(27,2 \%$; hombres: $22,3 \%$ ) y el drama $(22,6 \%$; hombres: $17 \%)$. No hay casi diferencias en el de animación (hombres: 5,8 \%; mujeres: 5,2 \%), algo en documentales (hombres: 7,8 \%; mujeres: $6,7 \%$ ), y bastantes en telenovelas (hombres: 0,5\%; mujeres: $2,1 \%$ ) (Tabla 1 ).

Tabla 1. Preferencias de contenidos visionados por género

\begin{tabular}{|l|l|l|l|}
\hline \multirow{2}{*}{\multicolumn{1}{c|}{ ¿\% columna) }} & \multicolumn{2}{c|}{ Sexo } & \multirow{2}{*}{ Total } \\
\cline { 2 - 3 } ¿Cuál es tu género favorito? & \multicolumn{1}{c|}{ Hombre } & Mujer & \\
\hline Acción & $33,0 \%$ & $17,6 \%$ & $21,1 \%$ \\
\hline Animación & $5,8 \%$ & $5,2 \%$ & $5,4 \%$ \\
\hline Comedia & $22,3 \%$ & $27,2 \%$ & $26,1 \%$ \\
\hline Documentales & $7,8 \%$ & $6,7 \%$ & $6,9 \%$ \\
\hline Drama & $17,0 \%$ & $22,6 \%$ & $21,3 \%$ \\
\hline Otros & $13,6 \%$ & $18,6 \%$ & $17,5 \%$ \\
\hline Telenovelas & $0,5 \%$ & $2,1 \%$ & $1,8 \%$ \\
\hline
\end{tabular}

Nos hemos preguntado si puede influir, si quiera aparentemente, el tipo de contenido audiovisual consumido en la visión del mundo, estimulando percepciones más o menos pesimistas 
proyectadas en la pandemia. Y efectivamente, los porcentajes de estudiantes que temen el contagio parecen variar según el género preferido. Los que menos temen son los que prefieren Animación (nada: $22,4 \%$ ), y los que más lo temen son los que prefieren documentales (9,5\%). Igualmente, Un $88,9 \%$ cree que habrá repuntes significativos este año, y entre ellos, lo que más lo creen son los que prefieren los documentales $(95,2 \%$ ) (Tabla 2$)$. Evidentemente, estos datos hay que tomarlos cum granum salis pero pueden avalar las hipótesis de la retroalimentación de los contenidos consumidos y la percepción de la realidad, la cual a su vez influye en las actitudes y decisiones.

Tabla 2. Temor al contagio según contenidos audiovisuales favoritos

\begin{tabular}{|l|c|c|c|c|}
\hline \multirow{2}{*}{$\begin{array}{l}\text { (\% fila) } \\
\text { ¿Cuál es tu género favorito? }\end{array}$} & \multicolumn{4}{|c|}{ ¿Tienes miedo a ser contagiado/a? } \\
\cline { 2 - 5 } & Nada & Poco & Bastante & Mucho \\
\hline Acción & $16,7 \%$ & $54,2 \%$ & $22,4 \%$ & $6,8 \%$ \\
\hline Animación & $22,4 \%$ & $63,3 \%$ & $12,2 \%$ & $2,0 \%$ \\
\hline Comedia & $14,7 \%$ & $53,8 \%$ & $23,9 \%$ & $7,6 \%$ \\
\hline Documentales & $14,3 \%$ & $44,4 \%$ & $31,7 \%$ & $9,5 \%$ \\
\hline Drama & $10,8 \%$ & $59,3 \%$ & $24,2 \%$ & $5,7 \%$ \\
\hline Otros & $22,0 \%$ & $54,7 \%$ & $22,0 \%$ & $1,3 \%$ \\
\hline Telenovelas & $18,8 \%$ & $43,8 \%$ & $31,3 \%$ & $6,3 \%$ \\
\hline Total & $16,0 \%$ & $54,9 \%$ & $23,4 \%$ & $5,7 \%$ \\
\hline
\end{tabular}

\section{CONCLUSIONES Y DISCUSIÓN}

Cabe suponer que los universitarios se vayan distinguiendo de los adolescentes en general, de forma positiva, con un ocio más cultural, pero de todas formas continúan acusando ciertos aspectos vulnerables. El hecho de vivir de forma independiente suele llevarlos a descuidar más la dieta ${ }^{16}$. El abuso de las pantallas también puede desviar un uso placentero y destinado a la autorrealización del tiempo libre hacia actitudes que podrían poner en situación de riesgos de diverso grado su bienestar.

Tanto de nuestro estudio como de otros similares se desprenden indicios que podrían ser preocupantes, que deben continuar siendo investigados, por lo tanto, y que asocian: 1) el alto consumo de contenidos de ficción al consumo social y por diversión del alcohol, 2) el alto consumo de videojuegos con los juegos de azar, una dieta no equilibrada y patrones disruptivos de los ritmos circadianos. Podría ser también interesante seguir la pista entre de la posible influencia del tipo de géneros consumidos en el miedo a los acontecimientos adversos y a la vida en general.

El confinamiento estricto no puede considerarse como un patrón de hábitos, pero puede servir de laboratorio para observar tendencias que proporcionen argumentos en los debates preparatorios de medidas de política social y educativa. La juventud universitaria siempre ha sido relativamente global, bien que ahora lo es en un sentido mucho más profundo y extenso. Las experiencias de intercambio cultural forman parte de las ventajas de ese rasgo, toda vez que fomentan el respeto por la diferencia. Sin embargo, la política de becas en España no ha acompañado en los últimos años la igualad de los estudiantes en este ámbito fundamental. La 


\section{EL BIENESTAR DE LOS UNIVERSITARIOS DURANTE EL CONFINAMIENTO POR COVID-19 FERNANDO GIL VILLA; JOSÉ DAVID URCHAGA LITAGO; ADRIÁN SÁNCHEZ FERNÁNDEZ}

actual crisis sanitaria añade un doble perjuicio. Por un lado, puede limitar la parte del presupuesto educativo dedicado al intercambio. Por otro, el confinamiento corta de raíz la vida cultural, lo cual nos permite neutralizar el valor de ese factor en la observación. Quedan los estudiantes, en estas condiciones, expuestos a la otra cara de la globalización, la de la interacción despersonalizada, a través de las nuevas tecnologías de la comunicación. Pero en este caso nos topamos con una limitación básica: la falta de alfabetización sanitaria -incluyendo la pedagogía de la muerte -y de educación para el ocio. Se trata de dos lagunas evidentes en nuestro sistema educativo que explican parte de nuestras dificultades para estar bien ${ }^{17}$. Ante esa ausencia, y con una pobre o nula experiencia intercultural, los jóvenes no están bien pertrechados para afrontar tragedias como la que supone la pandemia por COVID-19. A ello se suma la desafección política y religiosa, que en otros tiempos daba un sentido a la vida. La crisis sanitaria confirma, para el universitario y para el joven en general, la intuición de vivir en una sociedad incierta y vulnerable, propiciando más las sensaciones de angustia y malestar que las reacciones de serenidad, fortaleza, sacrificio y solidaridad, para las que no está preparado en general. Más bien se interna en un refugio individual con falsa apariencia social, completamente extemporáneo, fuera del llamado ético de los enfermos y más vulnerables: el mundo de las pantallas. Con ello sustituye la realidad por la ficción. En la medida en que niega la primera, contradice su papel de estudiante, que consiste en preguntarse por los problemas que lo rodean y reflexionar para ayudar a solucionarlos. Ciertamente, esta ruptura con la realidad, que en parte explicaría la existencia de quienes niegan la tragedia de la pandemia y hacen gala de dicha actitud, personas con títulos de educación superior, no se da en todos en el mismo grado. En general no puede decirse que es alarmante, pero sí preocupante. En un pequeño sector, además, aflora de tal perniciosa forma que requiere la atención de los expertos: allí donde se atenta abierta y largamente contra el reloj biológico y se acompaña de adicciones a sustancia psicoactivas -principalmente el alcohol-o de ludopatías no reconocidas.

El presente trabajo cobra sentido dentro de esta reflexión. Señala la necesidad realizar más estudios que pudieran corroborar y aclarar estas tendencias, así como, por otro lado, la urgencia de cambios en el sistema de enseñanza que mejoren la capacidad de resiliencia de los ciudadanos ante situaciones de emergencia como la actual.

\section{REFERENCIAS}

1. Brooks SK, Webster RK, Smith LE, Woodland, L., Wessely, S., Greenberg, N., et al. The psychological impact of quarantine and how to reduce it: rapid review of the evidence. Lancet. 2020;395(10227):912-20.

2. Wang C, Pan R, Wan X, Tan Y, Xu L, Ho CS, et al. Immediate psychological responses and associated factors during the initial stage of the 2019 Coronavirus Disease (COVID-19) Epidemic among the General Population in China. Int J Environ Res Public Health. 2020;17(5): 1729

3. Cao W, Fang Z, Hou G, Han M, Xu X, Dong J, et al. The psychological impact of the COVID-19 epidemic on college students in China. Psychiatry Res. 2020;287: 112934. /

4. Casagrande M, Favieri F, Tambelli R, Forte G. The enemy who sealed the world: effects quarantine due to the COVID-19 on sleep quality, anxiety, and psychological distress in the Italian population. Sleep Med. 2020;75:12-20.

5. Li S, Wang Y, Xue J, Zhao N, Zhu T. The impact of COVID-19 epidemic declaration on psychological consequences: a study on active weibo users. Int J Environ Res Public Health. 2020;17(6):2032.

6. Mezquita L, Stewart SH, Kuntsche E, Grant VV. Estudio transcultural del modelo de cinco factores de motivos de consumo de alcohol en universitarios españoles y canadienses. Adicciones. 2016;28(4):215-20. 


\section{EL BIENESTAR DE LOS UNIVERSITARIOS DURANTE EL CONFINAMIENTO POR COVID-19 FERNANDO GIL VILLA; JOSÉ DAVID URCHAGA LITAGO; ADRIÁN SÁNCHEZ FERNÁNDEZ}

7. Ballester-Arnal R, Gil-Llario MD. ¿Por qué los jóvenes se dan atracones de alcohol los fines de semana? estudio sobre creencias y actitudes relacionadas con este patrón de consumo y diferencias de género. RPPC. 2009; 14(1):25-35.

8. García-Álvarez L, Fuente-Tomás L, Sáiz PA, García-Portilla MP, Bobes J. ¿Se observarán cambios en el consumo de alcohol y tabaco durante el confinamiento por COVID-19?. Adicciones. 2020;32(2):85-9.

9. Margaritis I, Houdart S, El Ouadrhiri Y, Bigard X, Vuillemin A, Duché P. How to deal with COVID-19 epidemic-related lockdown physical inactivity and sedentary increase in youth? Adaptation of Anses' benchmarks. Arch Public Health. 2020;78(52).

10. Narici M, De Vito G, Franchi M, Paoli A, Moro T, Marcolin G, et al. Impact of sedentarism due to the COVID-19 home confinement on neuromuscular, cardiovascular and metabolic health: Physiological and pathophysiological implications and recommendations for physical and nutritional countermeasures. Eur J Sport Sci. 2020;1-22.

11. Orgiles M, Morales A, Delveccio E, Mazzeschi C, Espada JP. Immediate psychological effects of COVID-19 quarantine in youth from Italy and Spain (Consultado el 24 de abril de 2020).
12. Ballesteros JC, Megías E, Rodríguez E. Ocio y modelos de vida. La inevitable consolidación de las tecnologías en el tiempo libre de la juventud. Madrid: Centro Reina Sofía sobre Adolescencia y Juventud, Fad; 2020.

13. Rodríguez J, Agulló E. Estilos de vida, cultura, ocio y tiempo libre de los estudiantes universitarios. Psicothema. 1999;11(2):247-59.

14. Pollán-Santamaría M. Alimentación y cáncer: lo que hemos aprendido del estudio MCC-Spain. Ponencia Curso de verano Oncología Integrativa V. Pontevedra: Universidad de Santiago de Compostela. 2019.

15. Elche M, Sánchez-García S, Yubero S. Lectura, ocio y rendimiento académico en estudiantes universitarios del área socioeducativa. Educación XX1. 2019;22(1):215-37.

16. Chacón-Cuberos R, Castro-Sánchez M, Muros-Molina JJ, Espejo-Garcés T, Zurita-Ortega F, Linares-Manrique M. Adhesión a la dieta mediterránea en estudiantes universitarios y su relación con los hábitos de ocio digital. Nutr Hosp. 2016;33(2):405-10.

17. Gil-Villa F. Estar bien. Una reflexión desde la sociología clínica. Gijón: Sapere Aude; 2018. 


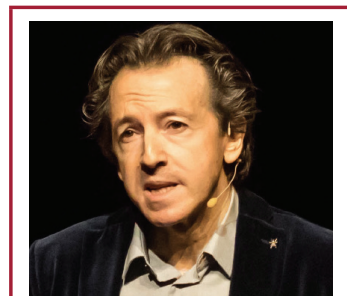

Fernando Gil Villa. Catedrático de Sociología de la Universidad de Salamanca. Cuatro tramos de Investigación y seis de docencia. Director del Grupo de Investigador Reconocido SEVIN (Sociedad, Educación y Violencia en la Infancia). Coordinador del Plan Estratégico de la USAL 2020-23. Profesor Visitante en la Universidad de Chicago, Portsmouth, Brasilia, etc. Líneas de investigación: sociología de la educación, de la salud y de la desviación.

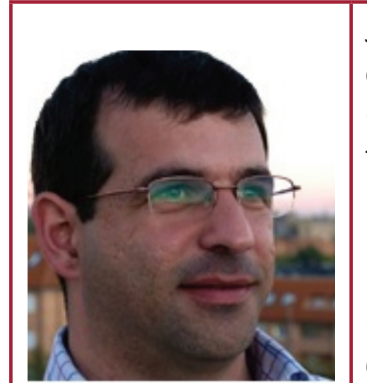

José David Urchaga Litago. Profesor de Metodología, Estadística y Psicología en la Facultad de Comunicación de la Universidad Pontificia de Salamanca (España). Áreas de interés: estadística multivariante, metodología cualitativa y cuantitativa, análisis de contenido de noticias y de publicidad, temas relacionados con hábitos en adolescentes y jóvenes (en especial los relacionados con salud, acoso escolar, valores, religiosidad, relaciones familiares, uso de medios, educación), desigualdad social, educación obligatoria y universitaria. Sexenio reconocido de investigación. Dirección de 21 tesis doctorales. Participación y dirección de varios proyectos de investigación financiados. Artículos en revistas indexadas en WOS o en Scopus: 17.

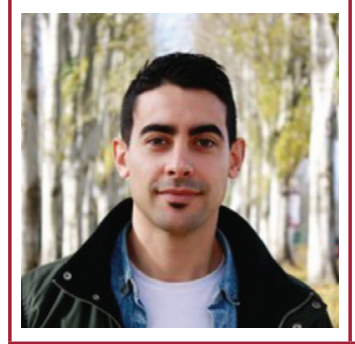

Adrián Sánchez-Fdez. Universidad de Salamanca. Licenciado en Biotecnología por la Universidad de Salamanca (España) y Bellarmine University (U.S.A). Máster en Biología y Clínica del Cáncer por la Universidad de Salamanca. Investigador predoctoral en el Instituto de Investigaciones Biomédicas de Salamanca (IBSAL) con financiación de la Asociación Española Contra el Cáncer (AECC). Miembro del equipo directivo de la Sociedad Española De Excelencia Académica (SEDEA) y de la European Association for Cancer Research (EACR). 